\title{
Microstructural characterisation of porous construction materials - major challenges
}

\author{
A. J. Klemm \\ Glasgow Caledonian University, UK
}

\begin{abstract}
This paper focuses on the microstructural characterisation of porous construction materials. The attempt was made here to assess the existing methods of analysis of pore structure in cementitious materials, such as Mercury Intrusion Porosimetry (MIP) and Differential Scanning Calorimetry (DSC). Due to a number of simplifying assumptions and limitations on the use of individual methods, it is not possible to describe in a qualitative, reliable way the microstructure of porous construction materials. The way to achieve this goal is to employ a wide range of complementary techniques.

Keywords: cementitious materials, microstructural characterisation, MIP, DSC, limitation of methods.
\end{abstract}

\section{Introduction}

The majority of materials in practice are or may be porous to a greater or lesser extent. Pores taking up the same relative volume in material may be characterised by a completely different structure (Fig. 1). They may exist in the form of closed pores, i.e. not connected with each other and not connected with the surface of material (for example some insulation materials made of foamed polymers) or in the form of less or more developed canals and gaps connected with each other and forming a network, penetrating the whole material evenly.

As the porosity structure greatly affects physical properties, strength characteristics and durability in general, a clear need is perceived to measure it.

Unfortunately, due to a number of simplifying assumptions and limitations on the use of individual methods, it is not possible to describe in a qualitative, reliable way the microstructure of porous construction materials. Although the Mercury Intrusion method is commonly regarded as the most convenient 
technique, mainly because it may be applied to moderately large samples $(1-3 g)$ and covers a very wide range of pore sizes $(100 \mu \mathrm{m}-3 \mathrm{~nm})$, its ability to yield accurate information about the size of the pores and their shape is very questionable. It is necessary to employ other techniques to assess errors in porosity analysis. Differential Scanning Calorimetry may become very useful here. This method allows detection of physical and chemical changes that are accompanied by a gain or loss of heat in a material as its temperature is increased or decreased. Characteristics of the phase transition process "water to ice" in porous materials may provide very important information.

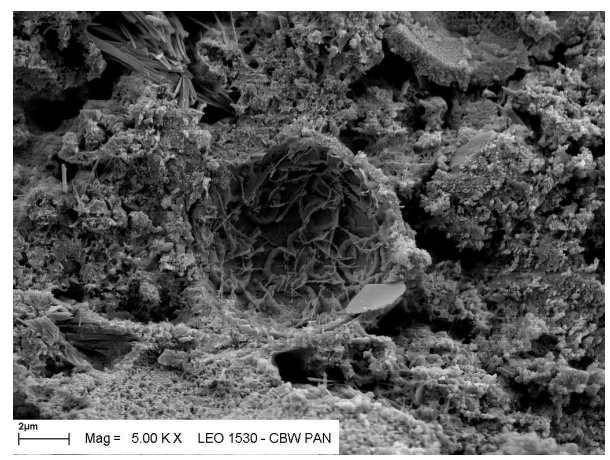

Figure 1: $\quad$ Microstructure of cement-based mortar.

\section{Differential Scanning Calorimetry}

Variations in the microstructure and chemical composition of materials result in substantial differences in the characteristics of phase transition in pores and capillaries. Generally capillaries, having the highest diameters, create the largest possibilities of spontaneous nucleus creation and then growth. The values of the supercooling, necessary to be reached in order to initiate the transition, are lower here. A more complex character of transition can be expected in smaller capillaries where the condition of ice penetration is the higher difference of the pressures between ice and water. This is again associated with the creation of internal forces acting on the composite matrix.

The Differential Scanning Calorimetry (DSC) technique can provide quantitative information about these changes by measuring the amount of heat that is involved as a material undergoes either an endothermic or exothermic transition. The gain (exothermic process) and loss (endothermic process) in the enthalpy of the material can be related to physical and chemical changes in the material during the heating or cooling.

Figure 2 shows an example of a DSC trace with three stages of phase transition clearly marked: "water to ice" - nucleus creation, spontaneous growth of nuclei and ice crystal growth.

As the temperature increases during heating, the 'onset', 'peak' and 'end' of any temperature endoderm are clearly distinguished so the heat of fusion in 
consequence can be calculated from the area encompassed. A similar procedure can be applied during cooling to obtain the location and magnitude of the temperature exotherm and again the heat of crystallization can be calculated from the area encompassed. Latent heats may be obtained by numerical integration of the area between the heat flow curve and the extrapolated base line. Melting and freezing points are taken at the intersection of the extrapolated base line and the tangent to the heat flow curve at the inflection point of the appropriate side of the peak. Melting and freezing 'peak' temperatures can be defined as the temperatures of the points that are located farthest from the base line in a heating or cooling cycle.

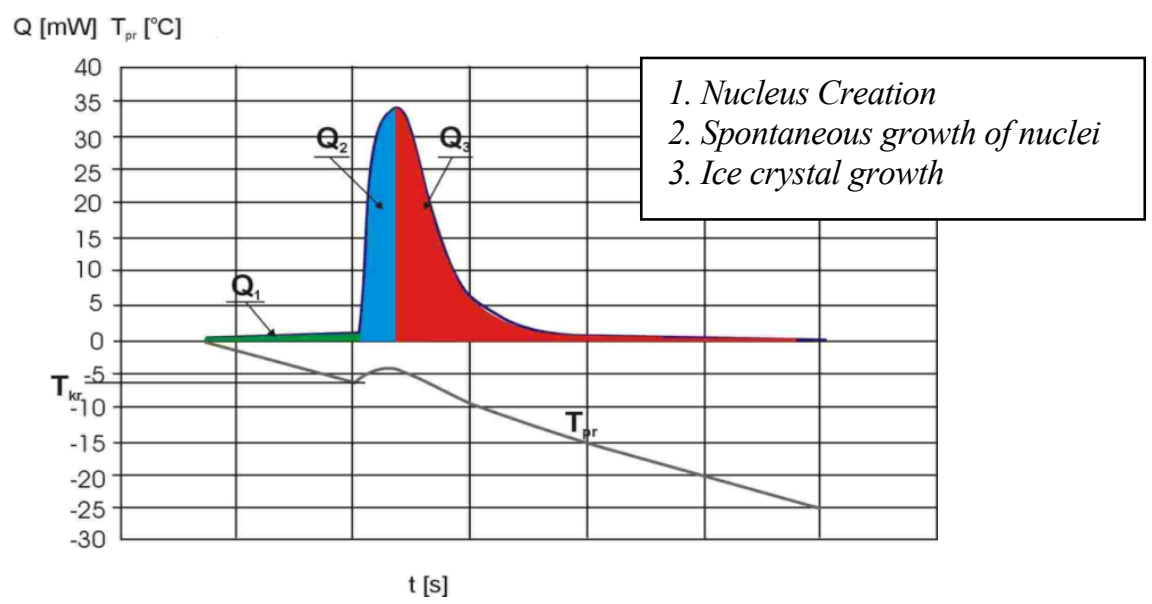

Figure 2: $\quad$ An example of a DSC trace.

Various environments (vacuum, inert atmosphere or controlled gas composition) and heating rates (from $0.1 \mathrm{~K} / \mathrm{min}$ to $320 \mathrm{~K} / \mathrm{min}$ ) can be employed for temperatures ranging from $-150^{\circ} \mathrm{C}$ to $2400^{\circ} \mathrm{C}$, depending upon the equipment used.

Considerable attention has been paid in the past to the influence of a number of factors - such as sample weight, particle size, heating and cooling rate, atmospheric conditions surrounding the sample - on the results obtained from DSC instruments. Whilst the influence of these should not be underestimated, the present design using milligram samples makes it relatively straightforward to obtain good results and reduces the effects of most of these factors.

Good technique is very necessary, and points such as possible misplacing of specimen dishes on the thermocouple platforms, inadequate drying of the sample or reference, selection of the wrong reference material, should not be overlooked.

\section{Mercury intrusion porosimetry}

Mercury porosimetry is the measuring method of open porosity. It allows not only determination of the total volume of open pores but also gives an 
information of the volume of pores having the specified sizes (pore size distribution). The Mercury Intrusion Porosimetry technique together with microscopic examinations and density measurements allows qualitative and quantitative description of porous material.

In principal a non-wetting liquid such as mercury penetrates a porous medium under gradually increasing pressure. The method of measurement is based on the capillary phenomenon. If a capillary having the radius, $r$, is placed in a container with liquid, the level of the liquid in such capillary depends on surface tension of liquid, $\gamma$ and the wetting angle of capillary material, $\theta$, by the liquid in container. In a case of the wetting angle in excess of $90^{\circ}$ the level of the liquid will eventually settle below the level of liquid in the container. In order to equalise the levels of liquid it is necessary to apply a pressure, $\mathrm{P}$, such as:

$$
\mathrm{P}=-2 \gamma \cos \theta / \mathrm{r}
$$

Since the different levels of pressure correspond with different radii of the penetrated pores, the volume of pores with radius between $r_{n-1}$ and $r_{n}$ can be easily determined. The number of measurements for increasing pressures allows the estimation of pore volumes as a function of their diameter, and the measurement of pore size distribution in the specimen tested. See example on Fig. 3 below.

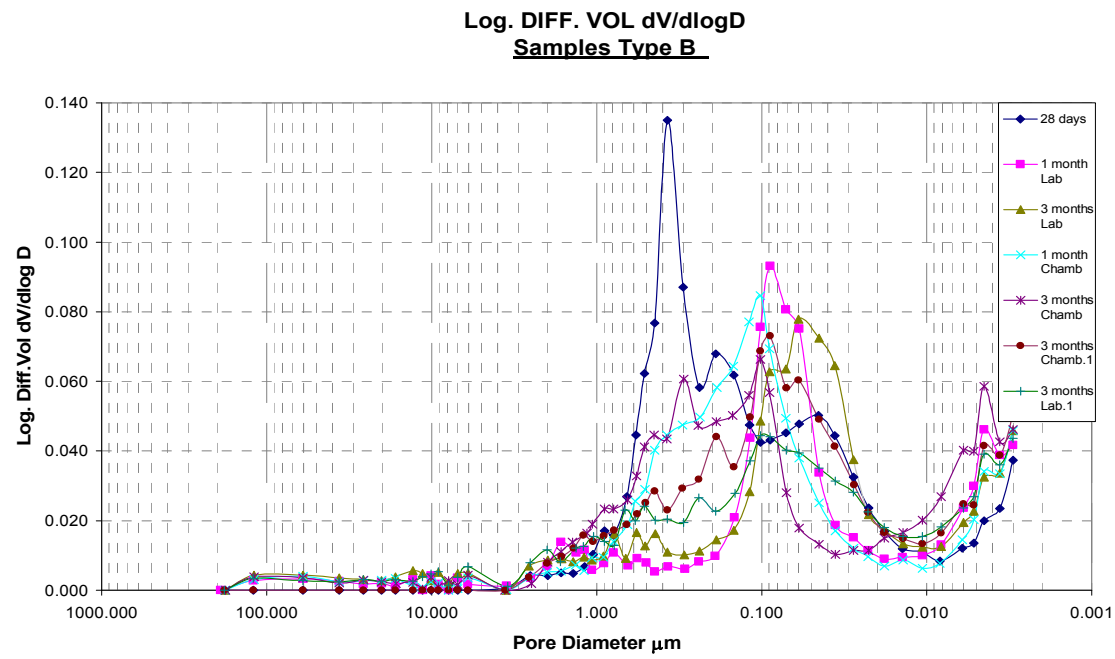

Figure 3: Example of a differential distribution curve.

Ability of the method to yield accurate information about the size of the pores and their shape depends on several factors and assumptions:

1. Compressibility of the solid, mercury, and residual air remaining in the sample space. All this factors may cause errors in the intrusion volume 
measurements. For some porous materials the compressibility may be higher because of the presence of open or closed pores.

2. Breakdown of porous materials under pressure. When mercury is forced into pores, the larger pores may be subject to breakdown, especially if they have bottleneck type constricted openings. For an equal wall thickness, the smaller pores are much stronger than the larger pores. Sponge or foam type materials are especially subjected to this type of "squeeze" effect because their walls are thin and of low mechanical strength.

3. Kinetic hysteresis (time effect) and volume hysteresis (pore shape effect). Sometimes considerable time is required for the mercury to flow into the pores at a given pressure. This time factor may be reduced or eliminated by increasing the time allowed for equilibrium. Volume hysteresis is the retention of mercury by the pores of the sample after penetration and reduction of pressure to one atmosphere (Fig. 4). The pore diameter calculated by the Washburn equation (1) is the diameter of the opening of the pores and not necessarily the largest diameter of the pore; the latter depends on the pore's shape.

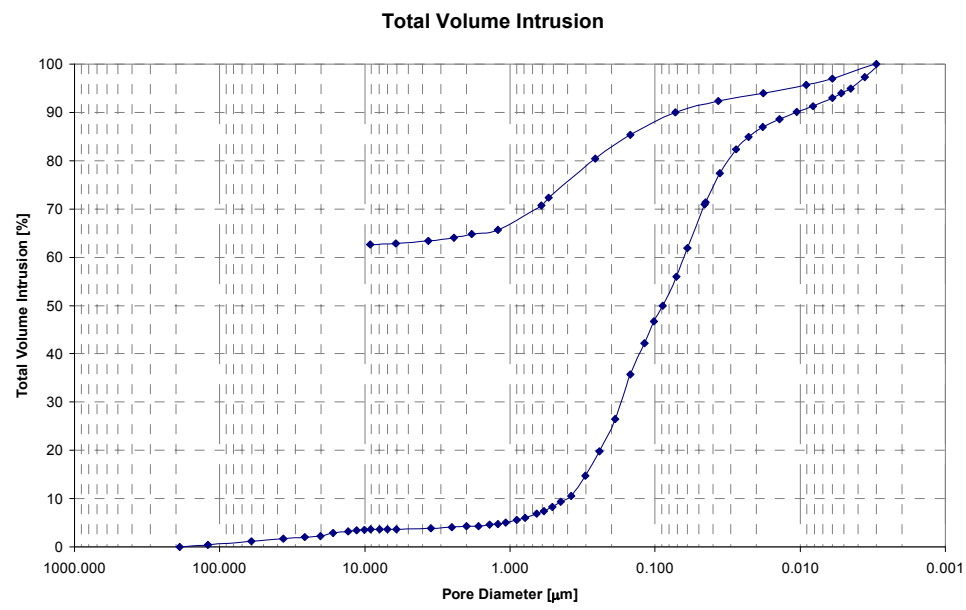

Figure 4: $\quad$ Example of a cumulative distribution curve.

4. Assumption of cylindrical pore model. The computations are based on a theoretical model in which the material is assumed to contain cylindrical and non-interacting pores. This is obviously not the case in practice.

5. Assumption of constant or given value for the surface tension of mercury. The degree of purity of mercury has a much greater effect on the surface tension of mercury, than the ambient temperature. Increase of contaminants will lower the surface tension of mercury and this may very well be the main factor in the wide spread of reported values for the surface tension of mercury in the literature. 
6. Assumption of some constant value for the contact angle between mercury and the solid. Since this information is not always readily available, most workers usually assume, for the cementitious materials, either $130^{\circ}$ or $140^{\circ}$, depending on their preference. For comparing porous materials of the same type, it does not really matter which value one chooses, unless an attempt is being made to obtain an exact measure of the pore openings.

\section{Experiment}

\subsection{Material tested}

The results presented in this report were obtained using polymer modified mortar with the cement/sand ratio $1: 1$ and $0.1 \%$ by weight MHEC - methyl hydroxyl ethyl cellulose. Samples were made in the form of prisms $50 \mathrm{~mm}$ by $50 \mathrm{~mm}$ by $200 \mathrm{~mm}$ and they were cured in their moulds for 24 hours before being demoulded. The samples were than stored in the normal laboratory conditions for 28 days before testing [1].

\subsection{Experiment}

The Differential Scanning Calorimetry was used to monitor the process of water to ice transition in polymer modified mortar. The experiment was carried out using DSC system - UNIPAN $605 \mathrm{M}$. The basic technical tolerances of the equipment used were: calorimetric accuracy $\pm 2 \%$, temperature accuracy $\pm 0.1 \mathrm{~K}$, scanning speed $0.1 \mathrm{~K} / \mathrm{min}-20 \mathrm{~K} / \mathrm{min}$, the range of applied temperature 113 $723 \mathrm{~K}$, the cooling rate $2 \mathrm{~K} / \mathrm{min}$. Measurements were carried out on minute samples of approximately 50 - 100mg weight which were extracted from the representative prisms. Specimens were saturated with water (48 hours) prior testing [2].

Mercury Intrusion Porosimetry method was used to determine total porosity, pore size distribution and total pore area. Small samples of about 2-3 g were tested in

Mercury Intrusion Porosimeter (Micromeritics AUTOPORE II 9220 V3.00).

\section{Experimental results}

\subsection{Differential Scanning Calorimetry}

Phase transition analyses were based on Everett [3] and the Everett and Hynes model [4]. The overall heat of phase transition, $\Delta \mathrm{Q}$ was determined to be equal to $0.96545 \mathrm{~J}$, leading to $18 \%$ increase in ice concentration. Heat emissions during three stages of phase transition i.e. nucleus creation, spontaneous growth of nuclei and growth of ice crystals, have been also separately estimated and presented in Tables 1- 3 .

The temperature in the chamber has been gradually lowered at a constant rate of $2 \mathrm{~K} / \mathrm{min}$. The value of the latent heat of solidification, $\Delta \mathrm{H}_{\mathrm{f}}$, has been assumed 
to be equal to $306.6 \mathrm{~J} / \mathrm{cm}^{3}$. The total volume of created ice has been estimated by using the following formula: $\Delta \mathrm{Q}=\Delta \mathrm{H}_{\mathrm{f}} \cdot \mathrm{V}_{\text {ice }}$

Figure 5 below shows heat evolution during three stages of phase transition.

Heat emission during the first stage of the phase transition for studied sample reached a value of $\mathrm{Q}_{\mathrm{I}}=0.00845 \mathrm{~J}$ (Table 1 ). During the first stage of transition, that value was equal to $\mathrm{V}_{\mathrm{I}}=2.75 \cdot 10^{-5} \mathrm{~cm}^{3}$ and the critical temperature of transition corresponding with the beginning of spontaneous growth of nucleus was $\mathrm{T}_{\mathrm{cr}}=268.4 \mathrm{~K}$. The value of supercooling $\Delta \mathrm{T}=\mathrm{T}_{\mathrm{cr}}-\mathrm{T}_{\mathrm{o}}\left(\mathrm{T}_{\mathrm{o}}=273 \mathrm{~K}\right)$ of water in pores and capillaries in a tested specimen was taken as equal to $\Delta \mathrm{T}=4.6 \mathrm{~K}$.

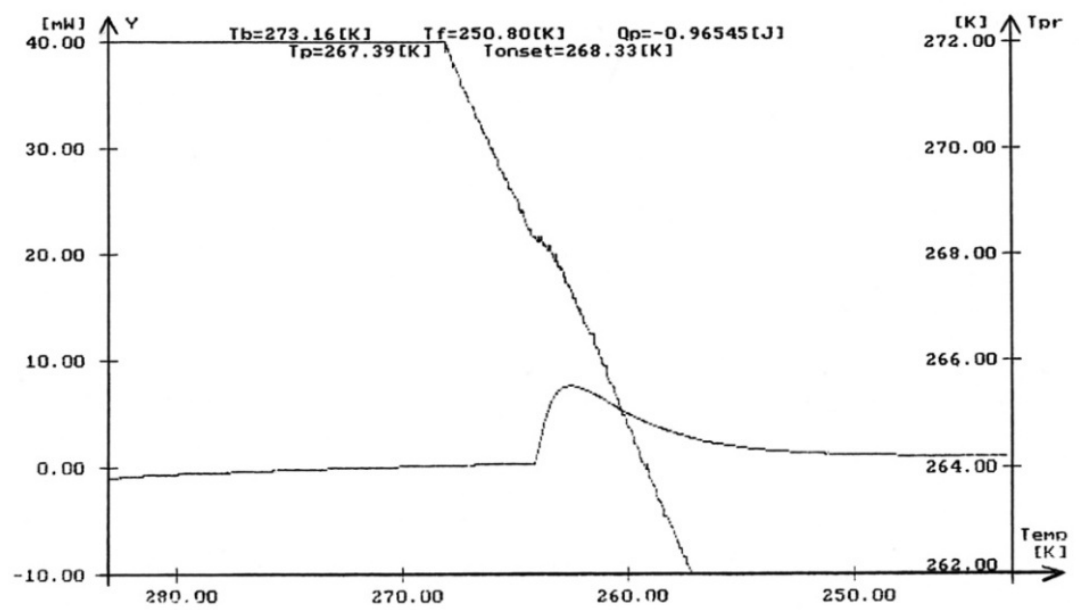

Figure 5: Differential Scanning Calorimetry curve.

Table 1: $\quad$ Stage I of transition - nucleus creation.

\begin{tabular}{|c|c|c|c|c|c|}
\hline \hline$\Delta \mathrm{Q}[\mathrm{J}]$ & $\Delta \mathrm{V}_{\mathrm{ice}}\left[\mathrm{cm}^{3}\right]$ & $\Delta \kappa[\%]$ & $\mathrm{T}_{\mathrm{cr}}[\mathrm{K}]$ & $\Delta \mathrm{T}[\mathrm{K}]$ & $\mathrm{r}_{\mathrm{cr}}[\mathrm{nm}]$ \\
\hline 0.0084 & $2.75 .10^{-5}$ & 0.2 & 268.4 & 4.6 & 541 \\
\hline
\end{tabular}

Where: $\Delta \mathrm{Q}$ - the heat of phase transition, $\Delta \mathrm{V}_{\text {ice }}$ - the volume of ice, $\Delta \kappa-$ the increment of ice concentration, $\Delta \mathrm{T}$ - the supercooling necessary to initiate the transition, $\mathrm{T}_{\mathrm{cr}}$ - the critical temperature, $\mathrm{r}_{\mathrm{cr}}$ - the critical radius of ice nucleus,

If the lower rate of the temperature decrease was assumed, which is a real condition, the value of supercooling would be much higher. At the critical temperature $\mathrm{T}_{\mathrm{cr}}=268.4 \mathrm{~K}$ nucleus or ice conglomerations, which are created in pores, reach their critical size. This quantity may be defined by using classical theory, which assumes the simplest way of nucleus creation i.e. when the nucleus 
stays in contact with their initial phase. The process of each phase creation is associated with the change of Gibbs energy $\Delta \mathrm{G}$, which in turn consists of two components: volumetric energy $\Delta \mathrm{G}_{\mathrm{v}}$ and superficial energy $\Delta \mathrm{G}_{\mathrm{s}}$. It is known, that if the temperature $T$ is lower than the temperature of solidification $T_{0}$, the change of free energy associated with the freezing per unit volume of liquid is equal to:

$$
\Delta \mathrm{G}_{\mathrm{v}}=\left(\Delta \mathrm{H}_{\mathrm{f}} / \mathrm{T}_{\mathrm{o}}\right) \Delta \mathrm{T}
$$

where: $\Delta \mathrm{H}$ is the latent heat of melting and $\Delta \mathrm{T}=\mathrm{T}_{\mathrm{o}}-\mathrm{T}$ is the supercooling.

Hence the change of thermodynamic potential accompanying the transition of volume from unstable to stable phase can be expressed by: $4 / 3 \pi r^{3} \Delta G_{v}$ and the superficial energy of the nucleus is defined as: $4 \pi \mathrm{r}^{2} \delta_{\text {iw }}$, where: $\delta_{\mathrm{iw}}$-the energy of the interphase is given as a function of the temperature.

During the phase transition the unstable phase with higher thermodynamic potential disappears and the new phase with lower potential is created. It is evident that the nuclei are stable when $\Delta \mathrm{G}$ is decreasing e.g. after reaching their critical size:

$$
\mathrm{r}_{\mathrm{cr}}=2 \delta_{\mathrm{iw}} / \Delta \mathrm{G}_{\mathrm{v}}
$$

The initiation of a spontaneous phase transition may occur when the nuclei of new phase reach their critical size. In a case of analyzed composite it can be presumed that critical radius of nucleus was equal to $r_{\mathrm{cr}}=541 \mathrm{~nm}$. That value has been obtained when $\Delta \mathrm{H}=306.6 \mathrm{~J} / \mathrm{cm}^{3}$ and $\delta_{\text {iw }}=250.10^{-6} \mathrm{~J} / \mathrm{cm}^{2}$ at $\mathrm{T}=268 \mathrm{~K}$ are assumed.

During the second stage of transition the significant amount of heat, which caused the temperature increment has been emitted (Table 2). The temperature increase caused by the phase transition was equal to $\Delta \mathrm{T}=1 \mathrm{~K}$ and the amount of liberated heat was $\mathrm{Q}_{\mathrm{II}}=0.1954 \mathrm{~J}$. Again the increment of ice volume in the second stage was $\Delta \mathrm{V}_{\mathrm{II}}=63.7 \cdot 10^{-5} \mathrm{~cm}^{3}$.

Table 2: $\quad$ Stage II of transition - spontaneous growth of nuclei.

\begin{tabular}{|c|c|c|c|c|c|}
\hline \hline$\Delta \mathrm{Q}[\mathrm{J}]$ & $\Delta \mathrm{V}_{\text {ice }}\left[\mathrm{cm}^{3}\right]$ & $\Delta \kappa[\%]$ & $\Delta \mathrm{T}[\mathrm{K}]$ & $\Delta \mathrm{p}\left[\mathrm{J} / \mathrm{cm}^{3}\right]$ & $\mathrm{R}_{\mathrm{cr}}[\mathrm{nm}]$ \\
\hline 0.1954 & $63.7 .10^{-5}$ & 3.6 & 5.6 & 6.29 & 795 \\
\hline
\end{tabular}

Where: $\Delta \mathrm{Q}$ - the heat of phase transition, $\Delta \mathrm{V}_{\text {ice }}$ - the volume of ice, $\Delta \kappa$ - the increment of ice concentration, $\Delta \mathrm{T}$ - the supercooling, $\Delta \mathrm{p}$ - the difference of pressures between ice and water $\left(\mathrm{p}_{\mathrm{W}}-\mathrm{p}_{\mathrm{i}}\right), \mathrm{R}_{\mathrm{c}}$ - the smallest radius of capillary in which ice is formed.

The pressure difference between water and ice inside pores could be estimated by using the following relationship:

$$
\left(\mathrm{p}^{*}-\mathrm{p}_{\mathrm{i}}\right)=\Delta \mathrm{S}_{\mathrm{f}}\left(\mathrm{T}-\mathrm{T}_{\mathrm{o}}\right)
$$

When the entropy of phase transition was assumed to be equal to $\Delta \mathrm{S}=1.123$ $\mathrm{J} / \mathrm{cm}^{3} \mathrm{~K}$ and the supercooling $\mathrm{T}-\mathrm{T}_{\mathrm{o}}=5.6 \mathrm{~K}$, the pressure difference was $\left(\mathrm{p}_{\mathrm{i}}-\mathrm{p}^{*}\right)=$ $6.29 \mathrm{~J} / \mathrm{cm}^{3}$. From the following relationship: 


$$
\mathrm{p}_{\mathrm{i}}-\mathrm{p}^{*}=2 \delta_{\mathrm{iw}} / \mathrm{R}_{\mathrm{c}}
$$

it was found that when $\left(p_{i}-p^{*}\right)=6.29 \mathrm{~J} / \mathrm{cm}^{3}$, ice penetration to capillaries having radius $R_{c}=795 \mathrm{~nm}$ can occur.

During the third stage of transition i.e. when the ice crystals started growing, the recorded amount of librated heat was $\mathrm{Q}_{\mathrm{III}}=0.76997 \mathrm{~J}$ and the corresponding increment of ice volume was $\Delta \mathrm{V}_{\mathrm{III}}=251 \cdot 10^{-5} \mathrm{~cm}^{3}$. This means, that after reaching a temperature $\mathrm{T}=250.8 \mathrm{~K}, 14.5$ per cent of water was involved with the phase transition in studied composite (Table 3 ).

\subsection{Mercury intrusion porosimetry}

Figure 6 presents the results of pore size distribution in mortar sample, clearly indicating the dominant range of pores between $700 \mathrm{~nm}$ and $2000 \mathrm{~nm}$.

It is evident that one of the most important factors in the application of mercury intrusion data is the value selected for the interfacial contact angle between the mercury and the solid. The better we can estimate the true contact

Table 3: $\quad$ Stage III of transition - ice crystal growth.

\begin{tabular}{||c|c|c|c|c|c||}
\hline$\Delta \mathrm{Q}[\mathrm{J}]$ & $\Delta \mathrm{V}_{\mathrm{ice}}\left[\mathrm{cm}^{3}\right]$ & $\Delta \kappa[\%]$ & $\Delta \mathrm{T}[\mathrm{K}]$ & $\Delta \mathrm{p}\left[\mathrm{J} / \mathrm{cm}^{3}\right]$ & $\mathrm{R}_{\mathrm{cr}}[\mathrm{nm}]$ \\
\hline 0.7699 & $251.10^{-5}$ & 14.5 & 22.2 & 24.93 & 281 \\
\hline
\end{tabular}

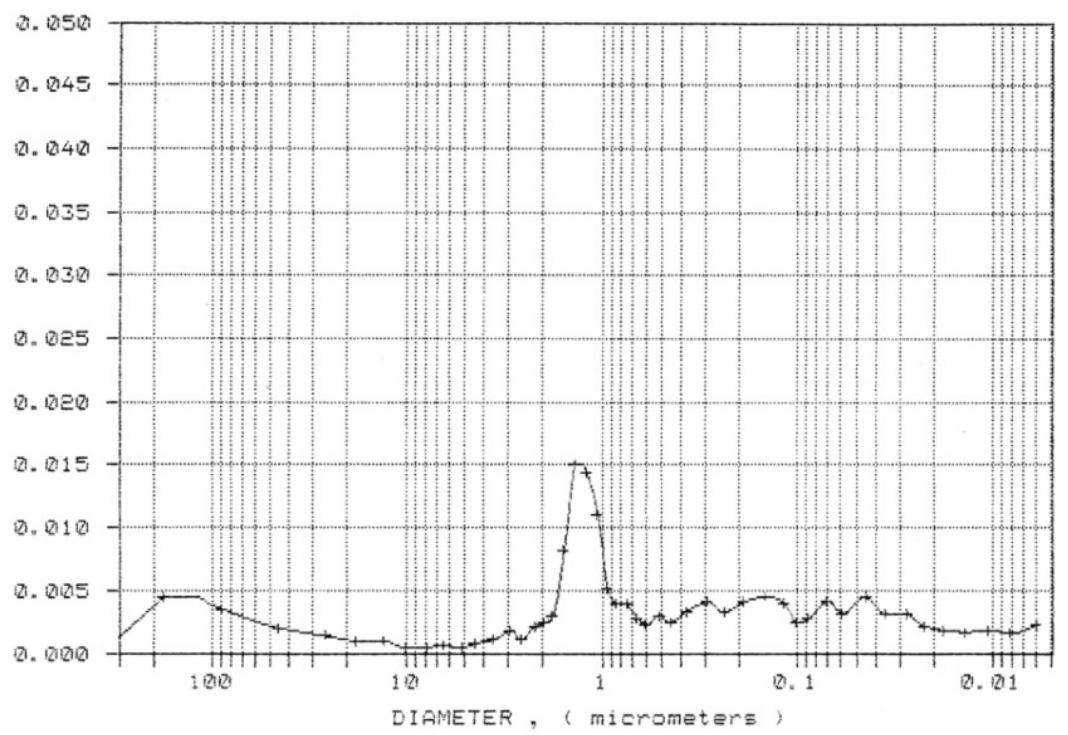

Figure 6: Differential distribution curve for the studied sample. 
angle value, the more accurate will be our subsequent calculations. The value of contact angle was assumed to be $\theta=130^{\circ}$ and surface tension of mercury $\gamma=$ 485 dynes $/ \mathrm{cm}$.

The cumulative distribution curve is shown on Fig 7. The following results were obtained: total porosity $0.148 \mathrm{~mL} / \mathrm{g}$, specific surface $2.843 \mathrm{~m}^{2} / \mathrm{g}$, median pore diameter (volume) $1.012 \mu \mathrm{m}$, threshold diameter $2.594 \mu \mathrm{m}$.

\subsection{Discussion}

Dominating range of pores in the MIP results lies between $700-2000 \mathrm{~nm}$.

On the other hand, results from DSC indicate the importance of $600-1600$ $\mathrm{nm}$. Beginning of the spontaneous nuclei growth $\left(2^{\text {nd }}\right.$ stage of transition) is associated with the increase of pressure between water and ice up to $6.29 \mathrm{~J} / \mathrm{cm}^{3}$ (912psi) and penetration of ice into pores of diameter 1590nm. Further increase of pressure up to $24.93 \mathrm{~J} / \mathrm{cm}^{3}$ (3618psi) leads to penetration of $560 \mathrm{~nm}$ pores and marks the beginning of the $3^{\text {rd }}$ stage of transition. Second stage of transition, which is mostly responsible for frost damage, mainly because of considerable increase of pressure over a relatively short period of time, is clearly placed in between these points. Higher volume of pores in this range (approximately 50\%) facilitated spontaneous ice growth.

Saturation of samples with water prior to the DSC testing could lead to rehydration and possibly to shifting dominant range of pores towards smaller pores.

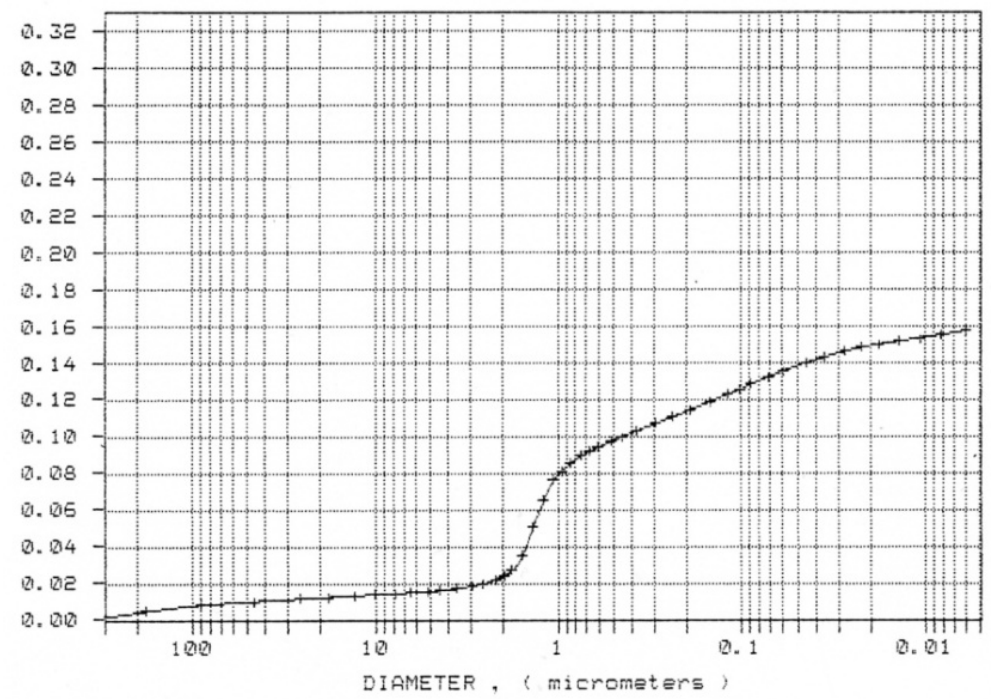

Figure 7: The cumulative distribution curve. 
Penetration of mercury under similar pressures leads to much smaller pores being filled in, correspondingly 200 and 50nm. This should be associated with differences in contact angle between mercury-solid and ice-solid. Variation of contact angle due to differences in chemical composition of composites may further complicate analysis.

\section{Summary}

High percentage of 'ink bottle' shaped pores is always shown by the hysteresis on the mercury intrusion curves. The release of pressure is not accompanied by expulsion of the same volume of mercury as was intruded as the pressure rose. This means that for the mercury volume corresponding with the difference of these values, the porosity model used for the specific surface determination from the porosimetric measurements is inappropriate. This model assumes that the mercury can penetrate into cylindrical shaped pores and capillaries by order of their sizes, starting from the biggest pores and ending with the smallest ones. For a medium with a microstructure satisfying this condition, during the pressure reduction the mercury does not 'tear away'. But if the penetrating mercury was intruded thought the 'inlet' into the larger pore it would be retained there. This means that the specific surface is calculated by the computer program with an error and indeed this value might be significantly lower than that from the test report. Unfortunately it is impossible even approximately to estimate this error. The method known from literature can only be applied to the specific samples which meet the condition mentioned above. At this stage an accurate estimation necessitate a change of the porosity model.

The complicated nature of pore structure causes difficulties since their sizes are categorised by only one geometric quantity i.e. the exit diameter of the pore. Nevertheless, it is assumed for analysis purposes, that the pores are open at both ends and have a cylindrical shape. Although according to Diamond [5], the failure of the MIP technique mostly originates from the accessibility effect rather than shape irregularities. It is due to the fact that the Washburn's relation Eq.1 assesses the dimension of a pore at a given pressure, on condition that mercury has accessed that pore. The interior of a sample is accessible only through a chain of pores varying in size and shape. Mature cement pastes contain a large number of randomly distributed segmented capillaries, which are interconnected solely through gel pores.

Another problem is associated with difficulties in determination of surface tension of mercury. One can attempt to correct for this by measuring the surface tension of mercury prior to using it, and after the run has been completed, determine if contamination of mercury in contact with the sample has occurred. This would result in a corresponding lowering of the surface tension.

It should be also stressed that if the samples differ in their chemistry changes in the contact angle should be expected. In such cases, the contact angle should be either measured directly or the pores examined under an optical or electron microscope, to establish the relationship for the actual pore size opening to that measured by mercury intrusion. Problem is even more complex if samples 
contain air entraining admixtures with the hydrophobic properties of entrained bubbles. Differences in contact angle in original pores and air entrained bubbles always result in an increased overall volume of pores of very low diameters. Estimation of contact angle is impossible by using standard techniques. It is therefore proposed to adopt more complex approach by simultaneous application of methods such as MIP, DSC and possibly SEM. Qualitative systematic analysis should enable estimation of an error made in pore size distribution measurements.

\section{References}

[1] Klemm A. J, Klemm P "Ice formation in pores in polymer modified concrete I. The influence of the admixture on the water to ice transition" Journal of Building and Environment, Vol.32, No 3, pp. 195-198 (1997)

[2] Klemm A. J, Klemm P. "Ice formation in pores in polymer modified concrete II. The influence of the admixtures on the water to ice transition in the cementitious composites subjected to freezing/thawing cycles” Journal of Building and Environment, Vol.32, No.3, pp. 199-202 (1997)

[3] Everett D.H., "Thermodynamics of Frost Damage to Porous Solids", Trans. Farad. Soc. Vol.56, pp.1541 -1551 (1961)

[4] Everett D.H., Hynes J.M., "Capillary Properties of Some Model Pore Systems with Special Reference to Frost Damage" Rilem Bull. No27, pp.31 $-38(1965)$

[5] Diamond S., "Mercury porosimetry. An inappropriate method for the measurement of pore distributions in cement-based materials." Cement and Concrete Research, Vol.30, pp.1517-1525 (2000) 